\title{
Successive sebopsoriasis, erysipelas and Koebner phenomenon
}

\author{
Manuelle Viguier MD PhD, Hervé Bachelez MD PhD
}

Previously published at www.cmaj.ca

$\mathrm{A}$ 48-year-old man presented with severe psoriasis vulgaris that was resistant to treatment and localized mainly in his limbs. He was given efalizumab. After 12 weeks, his initial skin lesions had regressed dramatically, but he had developed new facial seborrheic lesions (sebopsoriasis) (Figure 1A). Previously, his face had not been affected by a skin condition. Because we suspected that these new lesions were related to efalizumab therapy, we stopped the drug and substituted etanercept, a tumour necrosis factor $\alpha$ inhibitor.

After 15 days of treatment with
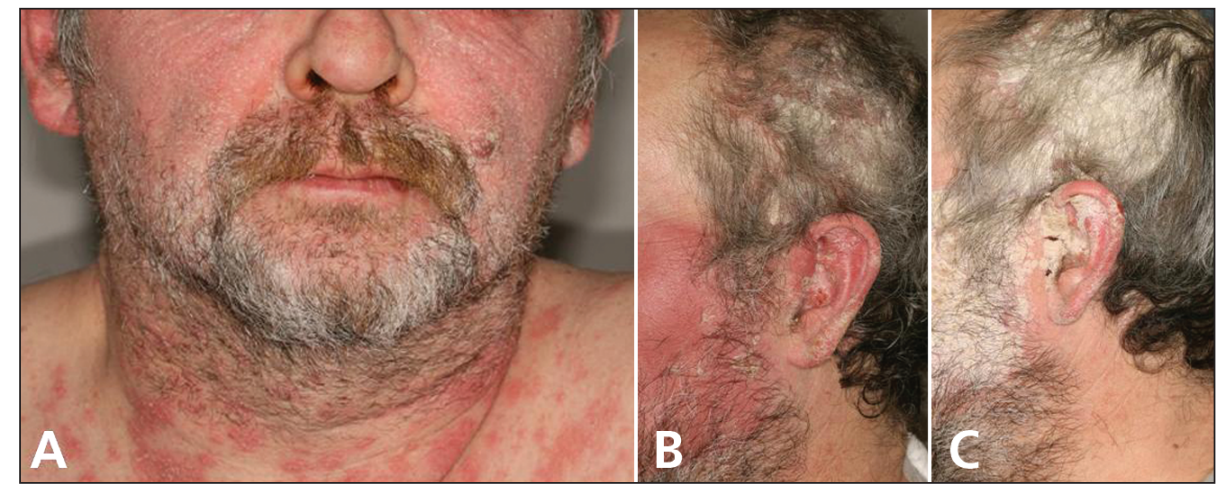

Figure 1: (A) Psoriasis on the face of a 48-year-old man with nonfacial psoriasis vulgaris, occurring during efalizumab therapy. (B) Facial erysipelas after replacement of efalizumab with etanercept. (C) Plaque psoriasis on the area previously affected with erysipelas, occurring after the withdrawal of etanercept. etanercept, the patient presented with fever, chills, malaise and an erythematous and edematous plaque with well-defined margins involving the left side of his face (Figure 1B). Facial erysipelas was diagnosed. The etanercept was stopped and pristinamycin was administered. Two weeks later, all signs of soft-tissue infection had resolved and been replaced by a typical psoriasis plaque at the site of previous erysipelas (Koebner phenomenon) (Figure 1C). We reintroduced etanercept, which led to a rapid remission of the plaque.

Efalizumab, a monoclonal antibody against the CD11a molecule, inhibits several steps in development of psoriasis. It had been approved for the treatment of moderate to severe psoriasis, but sales recently were suspended worldwide because of instances of progressive multifocal leukoencephalopathy.

Koebner phenomenon in psoriasis is the onset of psoriatic lesions on clinically uninvolved areas of skin in patients with

From the Department of Dermatology, Université Paris Diderot-Paris 7, and Hôpital Saint-Louis, Assistance publique-Hôpitaux de Paris, Paris, France

CMAJ 2010. DOI:10.1503/cmaj.091387 psoriasis that occurs as a result of trauma or infection. ${ }^{1}$ In our patient, the efalizumab-related sebopsoriasis was pruriginous, and subsequent skin erosion may have been the portal of entry for bacteria. Erysipelas, an acute infection of the dermis and hypodermis, is generally caused by group A streptococci. Treatment with tumour necrosis factor $\alpha$ antagonists has been associated with serious infections by mycobacteria, bacteria and fungi. ${ }^{2}$ In our patient, the withdrawal of etanercept because of acute infection was partially responsible for the development of the Koebner phenomenon. ${ }^{3}$

This article has been peer reviewed.

Competing interests: None declared.

\section{REFERENCES}

1. Weiss G, Shemer A, Trau H. The Koebner phenomenon: review of the literature. $J$ Eur Acad Dermatol Venereol 2002;16:241-8.

2. Smith CH, Anstey AV, Barker JNWN, et al. British Association of Dermatologists' guidelines for biologic interventions for psoriasis 2009. Br J Dermatol 2009;161;987-1019.

3. Carey W, Glaser S, Gottlieb AB, et al. Relapse, rebound, and psoriasis adverse events: an advisory group report. J Am Acad Dermatol 2006;54:S171-81. 